\title{
A Proposed Plan for Prenatal Care to Minimize Risks of COVID-19 to Patients and Providers: Focus on Hypertensive Disorders of Pregnancy
}

\author{
John R. Barton, MD, MS ${ }^{1}$ George R. Saade, MD $^{2}$ \\ ${ }^{1}$ Division of Maternal-Fetal Medicine,Baptist Health Lexington, \\ Lexington, Kentucky \\ 2 Division of Maternal-Fetal Medicine, University of Texas Medical \\ Branch, Galveston, Texas \\ ${ }^{3}$ Department of Obstetrics, Gynecology, Reproductive Sciences, \\ University of Texas-Houston, Houston, Texas \\ Am J Perinatol 2020;37:837-844.
}

\author{
Baha M. Sibai, MD³
}

Address for correspondence John R. Barton, MD, MS, Perinatal Diagnostic Center, 1740 Nicholasville Road, Lexington, KY 40503 (e-mail: jbarton@bhsi.com).

\begin{abstract}
Hypertensive disorders are the most common medical complications of pregnancy and a major cause of maternal and perinatal morbidity and death. The detection of elevated blood pressure during pregnancy is one of the cardinal aspects of optimal antenatal care. With the outbreak of novel coronavirus disease 2019 (COVID-19) and the risk for person-to-person spread of the virus, there is a desire to minimize unnecessary visits to health care facilities. Women should be classified as low risk or high risk for hypertensive disorders of pregnancy and adjustments can be accordingly made in the frequency of maternal and fetal surveillance. During this pandemic, all pregnant women should be encouraged to obtain a sphygmomanometer. Patients monitored for hypertension as an outpatient should receive written instructions on the important signs and symptoms of disease progression and provided contact information to report the development of any concern for change in status. As the clinical management of gestational hypertension and preeclampsia is the same, assessment of urinary protein is unnecessary in the management once a diagnosis of a hypertensive disorder of pregnancy is made. Pregnant women with suspected hypertensive disorders of pregnancy and signs and symptoms associated with the severe end of the disease spectrum (e.g., headaches, visual symptoms, epigastric pain, and pulmonary edema) should have an evaluation including complete blood count, serum creatinine level, and liver transaminases (aspartate aminotransferase and alanine aminotransferase). Further, if there is any evidence

Keywords

- preeclampsia

- hypertension in pregnancy

- coronavirus

- COVID-19 of disease progression or if acute severe hypertension develops, prompt hospitalization is suggested. Current guidelines from the American College of Obstetricians and Gynecologists (ACOG) and The Society for Maternal-Fetal Medicine (SMFM) for management of preeclampsia with severe features suggest delivery after $34^{0 / 7}$ weeks of gestation. With the outbreak of COVID-19, however, adjustments to this algorithm should be considered including delivery by $30^{0 / 7}$ weeks of gestation in the setting of preeclampsia with severe features.
\end{abstract}

\section{Key Points}

- Outbreak of novel coronavirus disease 2019 (COVID-19) warrants fewer office visits.

- Women should be classified for hypertension risk in pregnancy.

- Earlier delivery suggested with COVID-19 and hypertensive disorder.

received

April 9, 2020

accepted

April 15, 2020

published online

May 12, 2020
Copyright $\odot 2020$ by Thieme Medical Publishers, Inc., 333 Seventh Avenue, New York, NY 10001, USA. Tel: +1(212) 760-0888.
DOI https://doi.org/

10.1055/s-0040-1710538. ISSN 0735-1631. 
Hypertensive disorders are the most common medical complications of pregnancy and a major cause of maternal and perinatal morbidity and death. ${ }^{1}$ The detection of elevated blood pressure (BP) during pregnancy is one of the cardinal aspects of optimal antenatal care. Routine prenatal visits have therefore traditionally involved assessment of weight, $\mathrm{BP}$, urine protein by dipstick, and queries about symptoms suggestive of preeclampsia. With the outbreak of novel coronavirus disease 2019 (COVID-19) and the risk for person-to-person spread of the virus, there is a desire to minimize unnecessary visits to health care facilities. Since BP measurement is an essential component of prenatal care, and given that hypertension-related issues affect a considerable proportion of pregnant women, it is critical to develop a safe approach to reducing frequency of prenatal visits, as well as develop approaches to address women who are at home or quarantined, who may have hypertension or signs and symptoms associated with hypertensive disorders of pregnancy (HDP) that includes gestational hypertension (GHTN), preeclampsia, and superimposed preeclampsia.

In planning the frequency of prenatal visits, it is important to appreciate the rate of GHTN and preeclampsia, as well as the likely timing of onset of these complications, in the obstetrical population. At the first prenatal visit, each woman should be classified as low risk or high risk for HDP. In general, healthy nulliparous women and multiparous women with no prior history of HDP with a singleton gestation are considered low risk with an overall rate of HDP of $<10 \%$ (range: $2-5 \%$ for preeclampsia). ${ }^{2}$ Further, the onset of HDP in this group typically occurs at or beyond 37 weeks in approximately $75 \%$ of cases. In addition, the rate of HDP developing $<34$ weeks is $<1 \%{ }^{3}$ Consequently, prenatal visits can be safely reduced in such women prior to 36 weeks of gestation.

In contrast, women who are considered as high risk (those with previous history of HDP or with preexisting severe chronic hypertension) have a rate of HDP of 25 to $50 \%$, and the onset of these complications typically occurs at $<37$ weeks in approximately $50 \%$ of cases. ${ }^{4,5}$ Further, the rate of these complications developing at $<34$ weeks is approximately 10 to $15 \%$. A review of conditions with a high risk for developing hypertensive disorders of pregnancy ${ }^{5}$ are presented in -Table 1. Consequently, prenatal visits, as well as obstetric provider contact, should be more frequent in such women.

Table 1 Conditions with a high risk for developing hypertensive disorders of pregnancy

- Chronic hypertension/renal disease

- Pregestational diabetes mellitus

- Morbid obesity: BMI > $40 \mathrm{~kg} / \mathrm{m}^{2}$

- Systemic lupus erythematosus on medications (hydroxychloroquine plus steroids)

- Antiphospholipid antibody syndrome

- Prior pregnancy with preeclampsia at $<34$ weeks gestation

- Adverse outcome in a previous pregnancy (fetal growth restriction, abruption placentae, fetal death)
During this pandemic, all pregnant women should be encouraged to obtain a sphygmomanometer and bring the device to the office or clinic to ensure its accuracy. A device with an arm cuff is preferable; however, if these are unavailable, then a device with a wrist cuff is acceptable. If the patient cannot afford a sphygmomanometer or one is not provided by their insurance plan, then consideration can be given for BP determinations at the pharmacy. The important points for accurate clinical BP determination ${ }^{6}$ as an outpatient are reviewed in -Table 2.

\section{Management of Gestational Hypertension| Preeclampsia}

It is important to make decisions based on clinical judgment and common sense. Pregnant patients who are without known complications but have risk factors that require quarantine (recent travel to endemic areas or significant contact with an infected individual) and those with mild or asymptomatic COVID-19 infection should delay antenatal visits for 14 days.

The authors have previously reported that properly monitored outpatient management of nonsevere gestational hypertension/preeclampsia is associated with similar maternal and perinatal outcomes as compared with previously published results from inpatient management. ${ }^{7,8}$ Hospitalization, however, is recommended for noncompliant patients or those who show worsening course as outpatients. Pregnant women with suspected HDP due to hypertension and proteinuria or hypertension and signs and symptoms associated with the severe end of the disease spectrum (e.g., headaches, visual symptoms, epigastric pain, and pulmonary edema) should have an evaluation including complete blood count, serum creatinine level, and liver transaminases (aspartate aminotransferase and alanine aminotransferase $)^{9,10}$ Further, if there is any evidence of disease progression or if

Table 2 Important points for blood pressure measurement

- Patient should be seated comfortably with the back supported and upper arm bared without constrictive clothing, legs not crossed and the feet be on the floor

- The arm should be supported at heart level, the bladder of the cuff should encircle at least $80 \%$ of the arm circumference. It is preferable to always use the same arm for all blood pressure measurements (-Fig. 1A)

- Neither the patient nor the observer should talk during the measurement

- The position of the arm can have a major influence on the measured BP

- The patient should refrain from caffeine intake prior to BP measurements.

- If the upper arm is below the level of the right atrium (when the arm is hanging down while in the sitting position), the readings will be falsely high (-Fig. 1B)

- If the arm is above the heart level, the blood pressure readings will be falsely low (-Fig. 1B)

Note: Adapted from Pickering et al. ${ }^{6}$ 


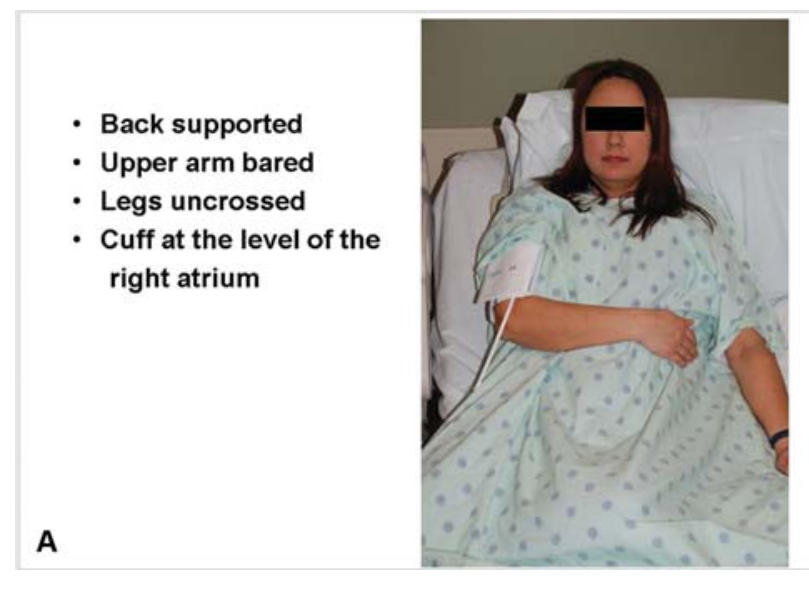

\section{Cuff above right atrial level \\ Artificially lower BP}

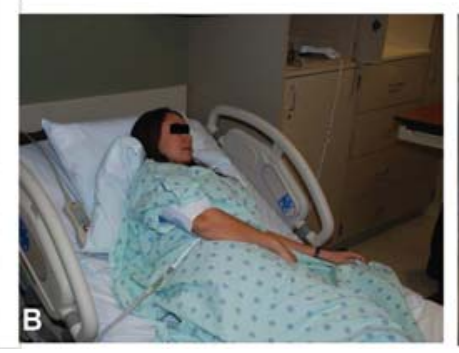

\section{Cuff below right atrial \\ level}

Artificially higher BP

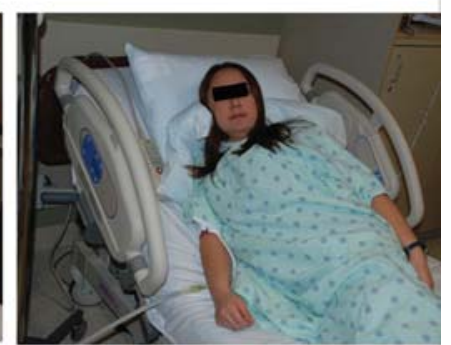

Fig. 1 (A) Proper position of the patient and blood pressure cuff for accurate blood pressure measurements. (B) Incorrect position of the patient and blood pressure cuff for accurate blood pressure measurements.

acute severe hypertension develops, prompt hospitalization is suggested.

Approximately $25 \%$ of women affected with HDP who develop one or more nonspecific symptoms that characterize the severe spectrum of the disease and signify the need for urgent evaluation and possible delivery, as described further.

\section{Persistent and/or Severe Headache}

The mechanism for headache, as well as other cerebrovascular symptoms of preeclampsia, is poorly understood. The headache is usually of acute onset and described as being the worst headache ever. The headache may be temporal, frontal, occipital, or diffuse. Although not pathognomonic, a feature that suggests preeclampsia-related headache rather than another type of headache is that it persists despite administration of over-the-counter analgesics such as acetaminophen in doses of $\leq 2 \mathrm{~g} /$ day. Concerning signs and symptoms relating to headache in pregnancy and postpartum include worsening headaches in women with chronic headaches; headache characteristic (e.g., pain and pattern) different from usual headaches; and headache with altered mental status, seizures, papilledema, changes in vision, stiff neck, or focal neurological signs or symptoms. ${ }^{11}$

\section{Visual Abnormalities (Scotomata, Photophobia, and Blurred Vision)}

Visual symptoms, when present, are also symptoms of the severe disease spectrum of preeclampsia. They are caused, at least in part, by retinal arteriolar spasm, macular edema, or vasogenic edema in occipital lobes.

\section{Upper Abdominal Epigastric Pain}

Gastroesophageal reflux is common in pregnant women, especially at night. Severe persistent right upper quadrant pain or epigastric pain unresponsive to medication and not accounted for by alternate diagnoses may be the presenting symptom of preeclampsia, particularly HELLP (haemolysis, elevated liver enzymes, low platelet count) syndrome. It is more significant when associated with abnormal liver enzymes.

\section{Cardiovascular Symptoms}

These include shortness of breath, retrosternal pressure or tightness in chest, dyspnea or orthopnea, and palpitations.

Patients monitored for hypertension as an outpatient should receive written instructions on the important signs and symptoms of disease progression and provided contact information to report the development of any concern for change in status. Sample instructions for home management of patients with gestational hypertension/preeclampsia ${ }^{12}$ are provided in - Table 3. Home BP assessment should be discussed. Methods by which BP measurements can be conveyed to the health care provider are provided in - Table 4. It is important to institute a process by which the appropriate provider is informed of the BP, particularly if outside a prespecified range, and that the information and interaction with the patient are documented in the medical record with suggestions for further BP determinations, timing of office visits, and confirmation that the patient remains a candidate for home management. The patient should be asked about the symptoms of severe preeclampsia at every encounter. It would be useful to have a check list that can be included in the record.

Blood Pressure Assessment Outline for Patients with Gestational Hypertension/Preeclampsia Managed at Home

- BP should be obtained thrice per day.

- The first BP assessment should be made in the morning before breakfast (especially before any caffeinated beverages).

- The second BP assessment performed 8 hours later.

- The third BP assessment performed before bedtime.

- Follow BP measurement guidelines as presented in -Table 2.

- BP measurements can be conveyed to the health care provider as outlined in -Table 4.

- If the systolic BP is $<150 \mathrm{~mm} \mathrm{Hg}$ and diastolic BP is $<100 \mathrm{~mm} \mathrm{Hg}$

- No change in frequency of measurement.

- Review symptoms of preeclampsia. 
Table 3 Sample instructions for home management of patients with HDP

\section{Contact your provider if}

- You have a severe, lasting headache that does not respond to extra strength acetaminophen (tylenol)

- You have pain in your abdomen, particularly around the stomach or the upper right area of the abdomen

- You noticed increased swelling of your face and around your eyes

- You have vaginal bleeding or spotting

- You notice fluid leaking from the vagina

- You experience regular uterine contractions or cramping

- You experience visual disturbances, such as seeing double, blurry vision, seeing floaters, or becoming very sensitive to light, numbness or altered mental status

- You experience shortness of breath, difficulty sleeping flat, chest pain or tightness

- You have any other symptom which cause you concern, such as persistent nausea, vomiting, or decreased fetal movement

- You should have been provided with a telephone number to reach the office or the labor and delivery unit to discuss your condition with the nurse or request that the physician be contacted. If you are unable to contact the physician or they do not return your call within 30 minutes or if your condition worsens within that time, go directly to the hospital, either by car or, if necessary, by ambulance

Abbreviation: HDP, hypertensive disorders of pregnancy. Note: Adapted from Barton et al. ${ }^{12}$

- If symptoms for severe features (severe lasting headache, right upper quadrant pain, and blurred or double vision) are present, then the patient should be instructed to go to the obstetrical triage or emergency room for further evaluation.

- If systolic BP is 150 to $159 \mathrm{~mm} \mathrm{Hg}$ or diastolic BP is 100 to $109 \mathrm{~mm} \mathrm{Hg}$

- Ensure that criteria in -Table 2 are met.

- Have patient rest for 15 minutes and repeat BP measurement.

- If repeat systolic BP is $<150 \mathrm{~mm} \mathrm{Hg}$ and diastolic BP is $<100 \mathrm{~mm} \mathrm{Hg}$, no change in frequency of measurement.

Table 4 Methods by which blood pressure measurements can be conveyed to the health care provider

- The patient may e-mail, text, video link, or phone the information to the office or clinic

- The patient may take a cell phone picture of blood pressure recordings on an electronic sphygmomanometer and forward this to the office or clinic

- Information can be relayed directly to the office or clinic via a mobile application
- If repeat BP remains in the same range, call the physician's office or the clinic, and review symptoms and disposition.

- If repeat systolic BP is $>160 \mathrm{~mm} \mathrm{Hg}$ or diastolic BP is $>110 \mathrm{~mm} \mathrm{Hg}$, instruct patient to go to the obstetrical triage or emergency room for further evaluation.

- If symptoms for severe features (severe lasting headache, right upper quadrant pain, and blurred or double vision) are present, then the patient should be instructed to go to the obstetrical triage or emergency room for further evaluation.

- If systolic BP is $\geq 160 \mathrm{~mm} \mathrm{Hg}$ or diastolic BP is $\geq 110 \mathrm{~mm} \mathrm{Hg}$

- Ensure that criteria in -Table 2 are met.

- Have patient rest for 15 minutes and repeat the BP measurement.

- If repeat systolic BP is $<150 \mathrm{~mm} \mathrm{Hg}$ and diastolic BP is $<100 \mathrm{~mm} \mathrm{Hg}$, no change in frequency of prenatal measurements.

- If repeat systolic BP is 150 to $159 \mathrm{~mm} \mathrm{Hg}$ or diastolic $\mathrm{BP}$ is 100 to $109 \mathrm{~mm} \mathrm{Hg}$, call the physician's office or the clinic and review symptoms and disposition. The patient should be seen in the office within 48 hours.

- If repeat $\mathrm{BP}$ remains $\geq 160 \mathrm{~mm} \mathrm{Hg}$ or diastolic BP $\geq 110 \mathrm{~mm} \mathrm{Hg}$, instruct patient to go to the obstetrical triage or emergency room for further evaluation.

- If symptoms for severe features (severe lasting headache, right upper quadrant pain, and blurred or double vision) are present, then the patient should be instructed to go to the hospital obstetric triage or emergency room for further evaluation.

\section{Management of Chronic Hypertension}

Chronic hypertension affects up to 5\% of pregnancies depending on the population studied, and its prevalence has risen over the past decade due to various factors including delayed childbearing, increasing prevalence of obesity, and increasing number of pregnancies with significant medical comorbidities such as pregestational diabetes, lupus, and renal disease. ${ }^{1}$ As previously noted, each woman should be classified as low risk or high risk for HDP at the first prenatal visit. - Table 5 compares the risk of adverse maternal and perinatal outcomes between women with low-risk chronic hypertension versus those who are high risk. The decision to treat BP should consider the presence of maternal comorbidities, as well as level of maternal BP, at the time of initial visit. There is general agreement that women with comorbidities (diabetes mellitus, renal disease, or heart disease) and those with severe hypertension (systolic BP $>160 \mathrm{~mm}$ $\mathrm{Hg}$ or diastolic $\mathrm{BP}>105 \mathrm{~mm} \mathrm{Hg}$ ) require antihypertensive therapy to keep maternal BP at a safe target range (systolic BP $<140 \mathrm{~mm} \mathrm{Hg}$ and diastolic BP $<90 \mathrm{~mm} \mathrm{Hg}$ ). ${ }^{1}$ In contrast, there is uncertainty regarding the optimal target goal BP to achieve in women with uncomplicated nonsevere hypertension during pregnancy.

In general, the frequency of evaluation for women with low-risk chronic hypertension can be similar to those of normotensive pregnancies. This management may be 


\begin{tabular}{|c|c|c|}
\hline Adverse outcome & $\begin{array}{l}\text { Low-risk CHTN } \\
\text { rate }(\%)\end{array}$ & $\begin{array}{l}\text { High-risk CHTN } \\
\text { rate (\%) }\end{array}$ \\
\hline $\begin{array}{l}\text { Superimposed } \\
\text { preeclampsia }\end{array}$ & $10-15$ & $30-50$ \\
\hline $\begin{array}{l}\text { Exacerbation to severe } \\
\text { hypertension }\end{array}$ & $7-10$ & $20-40$ \\
\hline Preterm delivery & $7-10$ & $20-50$ \\
\hline Fetal growth restriction & $10-12$ & $15-30$ \\
\hline Placental abruption & $1-2$ & $3-10$ \\
\hline Perinatal death & $<1$ & $3-15$ \\
\hline Renal Failure/dialysis & $<1$ & $1-2$ \\
\hline Retinal injury/stroke & $<1$ & $0.5-1$ \\
\hline Maternal death & $\begin{array}{l}\text { Exceedingly } \\
\text { rare }\end{array}$ & $0.5-1$ \\
\hline
\end{tabular}

Abbreviation: $\mathrm{CHTN}$, chronic hypertension. Note: Adapted from Chahine and Sibai. ${ }^{13}$

modified in instances where BP control requires medical intervention or fetal testing warrants closer follow-up. Such circumstances include exacerbation of hypertension requiring treatment (systolic BP $>160 \mathrm{~mm} \mathrm{Hg}$ and/or diastolic BP $>105 \mathrm{~mm} \mathrm{Hg}$ ), fetal growth restriction (estimated fetal weight $<10$ th percentile for gestational age with abnormal umbilical artery Doppler findings), or oligohydramnios (deepest vertical pocket $<2 \mathrm{~cm}$ ). ${ }^{13}$

Women with high-risk chronic hypertension require more intensive follow-up, given the increased risk in adverse maternal and perinatal outcomes and risk of further organ deterioration. In addition to the management of hypertension, any comorbid medical conditions should be adequately controlled, as pregnancy may aggravate preexisting condi- tions. Liberal hospitalization to manage these morbidities may be needed. ${ }^{13}$

\section{Blood Pressure Assessment: Outline for Patients with Chronic Hypertension}

- Blood pressure should be obtained twice per day

- The first BP assessment should be performed at 8 to 9 a.m. (especially before any caffeinated beverages).

- The second BP assessment should be performed at 8 to 9 p.m.

- Follow blood pressure measurement guidelines as presented in - Table 2.

- Blood pressure measurements can be conveyed to the health care provider as outlined in -Table 4.

Instructions for response to blood pressure measurements are as above for patients with gestational hypertension/ preeclampsia. In the setting of chronic hypertension with the patient receiving antihypertensive therapy, however, consideration could be given to increasing the antihypertensive medication dose.

\section{Besides BP Management in Patients with Hypertensive Disorders}

Given the limited information regarding the outcome of pregnancies complicated by COVID-19 infection, it is important to continue to use clinical judgment in obstetrical management during this pandemic. If concerned, or believe that the patient needs closer follow-up, she should be scheduled accordingly. - Table 6 presents suggested antepartum and postpartum guidelines for the management of patients with concern for HDP. - Table 7 presents suggested timing of delivery during the COVID-19 pandemic for patients with chronic hypertension, categorized by the classification of their chronic hypertension

Table 6 Antenatal management

1. Urine dipsticks

(a) To reduce frequency of prenatal visits and potentially health care interactions with patients with COVID-19, routine urine dipsticks do not need to be performed. As the clinical management of gestational hypertension and preeclampsia are the same, assessment of urinary protein is unnecessary in the management of HDP

(b) Send protein/creatinine ratios for the following

(i) Women with new elevations in BP and no history of hypertension (or send to obstetrical triage if appropriate)

(ii) Women with CHTN and new elevations in BP above their previous baseline

(c) Send urinalysis/urine culture for the following:

(i) Women with urinary tract infection (UTI) symptoms

(ii) New OB visit

2. Fetal testing

Ultrasound evaluations

Ultrasound is indicated to evaluate the amniotic fluid volume and estimate the fetal weight with the initial diagnosis of gestational hypertension or preeclampsia given the increased risk for oligohydramnios and fetal growth restriction in these patients. This is particularly true for preeclampsia that develops remote from term ${ }^{14}$ due to reduced uteroplacental perfusion, but less likely in those patients with a diagnosis made near term 
Table 6 (Continued)

The goal is to employ the maximum interval for follow up growth ultrasounds. Further, an attempt should be made to combine imaging with a prenatal visit in the ultrasound suite to avoid waiting in a second waiting room and seeing another provider team, thereby reducing contact with the potentially infected individual

(a) While the patient is in the ultrasound suite

(i) Medical assistants should take vital signs prior to starting the ultrasound

(ii) Medical providers can conduct a prenatal visit during the ultrasound

(iii) No urine dipsticks should be performed except for those with UTI symptoms

(b) Timing of follow up ultrasounds

(i) For GHTN/preeclampsia without severe features, ultrasound for growth every 4 weeks

(ii) For well controlled CHTN not requiring antihypertensive medication, ultrasound at 32-34 weeks with repeat evaluation 4 weeks later

(iii) For well controlled CHTN requiring antihypertensive medication, ultrasound at 32-34 weeks with repeat evaluation every 3 weeks

(iv) For poorly controlled CHTN requiring antihypertensive medication, ultrasound at 26 weeks with repeat evaluation every 3 weeks

(v) Avoid follow-up ultrasound for borderline AFI. Consider follow-up only when AFI is $5-6 \mathrm{~cm}$ and the follow-up should be in 1 week and not sooner (especially if MVP is $>2 \mathrm{~cm}$ )

\section{Antepartum fetal monitoring}

There are no data from randomized trials on which to base recommendations for the optimal type and frequency of antepartum fetal monitoring. However, daily fetal movement counts seem prudent. Weekly nonstress testing plus assessment of amniotic fluid volume, or weekly biophysical profiles should be performed at the time of diagnosis of GHTN/preeclampsia and continue until delivery. Fetal testing should promptly be performed if there is an abrupt change in maternal condition

\section{Expectant management and timing of delivery in HDP}

The main objective of the management of severe preeclampsia must always be the safety of the mother and the fetus. Although delivery is always appropriate for the mother, it might not be best for a very premature fetus. The decision between delivery and expectant treatment should take into consideration fetal gestational age, fetal status, and severity of maternal condition at the time of assessment including criteria used to make the diagnosis. The presence of severe preeclampsia mandates immediate hospitalization in the labor and delivery unit. ${ }^{9}$ After initial clinical and laboratory evaluation, a decision must be made for immediate delivery vs expectant treatment. Current guidelines from ACOG and SMFM for management of severe preeclampsia suggest delivery after 34.0 weeks gestation. With the outbreak of COVID-19, however adjustments to this algorithm should be considered including delivery by $30^{0 / 7}$ weeks of gestation in the setting of severe preeclampsia (-Fig. 2)

\section{Magnesium Sulfate Use}

Magnesium sulfate is a widely used medication in labor and delivery. It is recommended for seizure prophylaxis in patients with severe features of preeclampsia. ${ }^{10}$ However, magnesium sulfate therapy is not without risks. One major, albeit uncommon, side effect is respiratory depression. This can have serious implications for maternal outcomes, particularly for persons under investigation and known COVID-19-positive patients with an already compromised respiratory status. For patients with preeclampsia or gestational hypertension without severe features, magnesium sulfate therapy should not to be administered. ${ }^{1}$ In those with severe features, however, magnesium sulfate prophylaxis is indicated. As suggested by the results of the Magpie trial, ${ }^{15}$ an IV (intravenous) loading dose of $4 \mathrm{~g}$ magnesium sulfate over 15-20 min followed by an IV maintenance dose of $1 \mathrm{~g} / \mathrm{h}$ could be used rather than a traditional higher loading $(6 \mathrm{~g})$ and maintenance doses $(2 \mathrm{~g} / \mathrm{h})$. Patients with severe features of preeclampsia and requiring ventilatory support given COVID-19 infection should continue to receive intravenous magnesium sulfate for antiseizure prophylaxis as the respiratory rate can be adjusted with the settings of the mechanical ventilator.

6. Postpartum management up to 6 weeks

Comprehensive counseling about the signs/symptoms of preeclampsia should be given on discharge following delivery

(a) For patients with a BP cuff

(i) Schedule a telehealth visit 3-5 days after discharge.

(ii) Use video to view the BP measurement and display, or have the patient send a picture of the screen

(b) For patients without a BP cuff

(i) Schedule an in-person clinic visit 3-5 days after discharge.

(c) If comorbidities (CHTN, etc.)

(i) If hypertensive and has BP cuff, schedule phone visit at 3-5 days after discharge with clinic visit at 2 weeks

(ii) If hypertensive and no BP cuff, clinic visit in 3-5 days after discharge

(iii) Use clinical judgment as to frequency of visit and whether visit is by phone or in person

Abbreviations: ACOG, American College of Obstetricians and Gynecologists; AFI, amniotic fluid index; BP, blood pressure; CHTN, chronic hypertension; COVID-19, novel coronavirus disease 2019; GHTN, gestational hypertension; HDP, hypertensive disorders of pregnancy; MVP, maximum vertical pocket; SMFM, Society for Maternal-Fetal Medicine. 


\section{Severe Preeclampsia $\leq 30^{0 / 7}$ wks* $^{*}$ \\ *(during COVID-19 pandemic)}

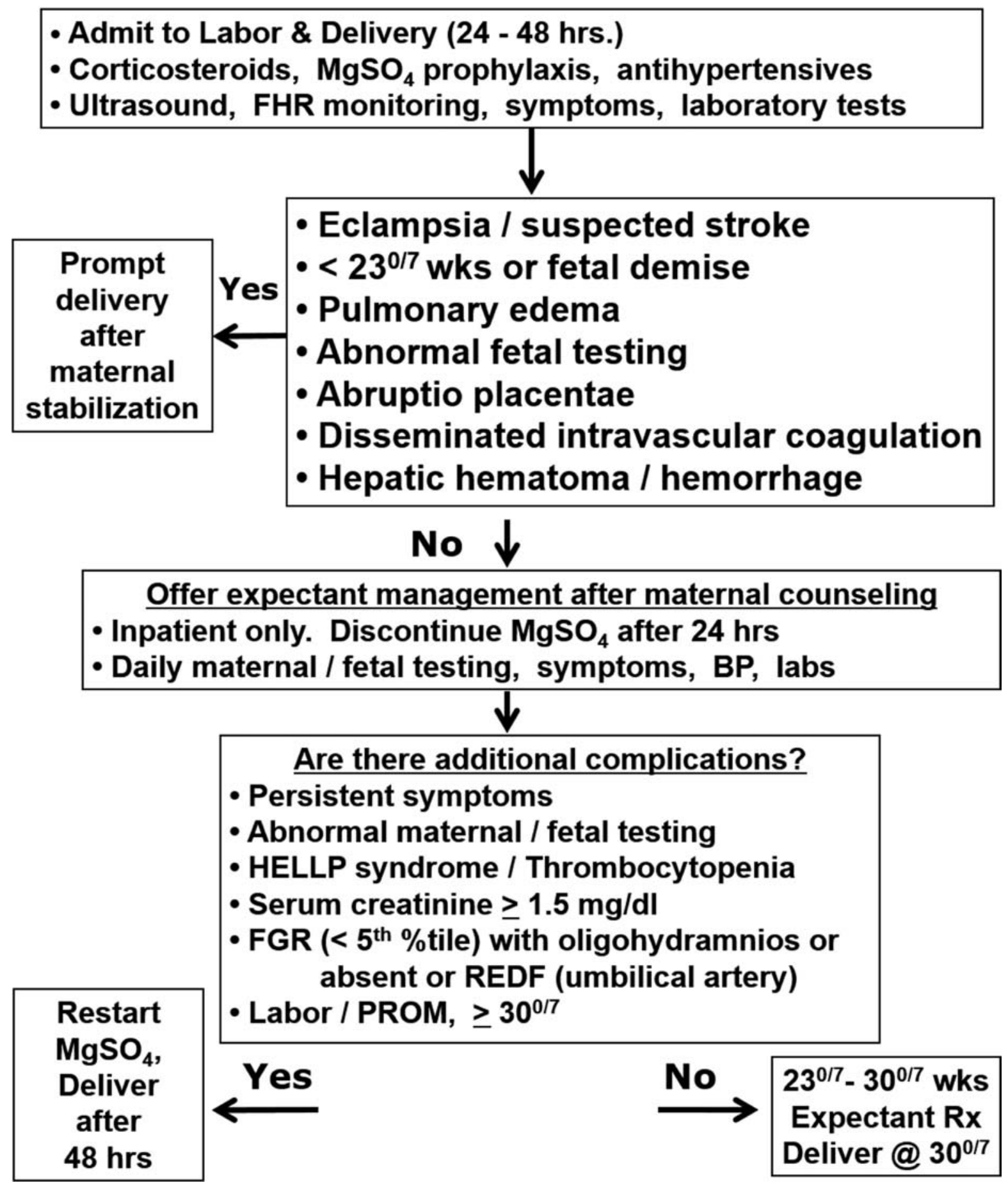

Fig. 2 Management of preeclampsia with severe features $\leq 30$ weeks of gestation during the COVID-19 pandemic. BP, blood pressure; COVID19, novel coronavirus disease 2019; FGR, fetal growth restriction; FHR, fetal heart rate; HELLP, haemolysis, elevated liver enzymes, low platelet count; REDF, reverse end-diastolic flow. 


\begin{tabular}{|c|c|}
\hline Clinical situation & $\begin{array}{l}\text { Gestational } \\
\text { age (wk) }\end{array}$ \\
\hline \multicolumn{2}{|l|}{ Low-risk chronic hypertension } \\
\hline Controlled without any medication & 39 \\
\hline Controlled with a single medication & 38 \\
\hline $\begin{array}{l}\text { With superimposed preeclampsia } \\
\text { without severe features }\end{array}$ & 37 \\
\hline $\begin{array}{l}\text { With isolated fetal growth restriction } \\
\text { (EFW }<10 \text { th percentile) or oligohydram- } \\
\text { nios (DVP }<2 \mathrm{~cm} \text { ) }\end{array}$ & 37 \\
\hline $\begin{array}{l}\text { With fetal growth restriction (EFW }<10 \text { th } \\
\text { percentile) and oligohydramnios and/or } \\
\text { abnormal umbilical artery Doppler }\end{array}$ & $32-36^{a}$ \\
\hline \multicolumn{2}{|l|}{ High-risk chronic hypertension } \\
\hline With pregestational diabetes & 36 \\
\hline $\begin{array}{l}\text { Controlled with maximum doses of two } \\
\text { medications }\end{array}$ & 36 \\
\hline $\begin{array}{l}\text { Controlled with maximum doses of three } \\
\text { medications }\end{array}$ & 34 \\
\hline $\begin{array}{l}\text { Uncontrolled/difficult to control blood } \\
\text { pressure }\end{array}$ & 34 \\
\hline $\begin{array}{l}\text { With superimposed preeclampsia with } \\
\text { severe features }\end{array}$ & 32 \\
\hline $\begin{array}{l}\text { With fetal growth restriction (EFW }<10 \text { th } \\
\text { percentile) and oligohydramnios and/or } \\
\text { abnormal umbilical artery Doppler }\end{array}$ & $32-34^{\mathrm{a}}$ \\
\hline With evidence of placental abruption & At diagnosis \\
\hline
\end{tabular}

Abbreviations: COVID-19, novel coronavirus disease 2019; EFW, estimated fetal weight; DVP, deepest vertical pocket. Note: Adapted from Chahine and Sibai. ${ }^{13}$

${ }^{\mathrm{a}} \mathrm{Gestational}$ age at delivery will depend on severity of fetal growth restriction and degree of changes in umbilical artery Doppler.

\section{Conclusion}

With the outbreak of COVID-19 and the risk for person-toperson spread of the virus, there is a desire to minimize unnecessary visits to health care facilities. Pregnant patients who are without known complications but have known risk factors for COVID 19 (recent travel to endemic areas or significant contact with an infected individual) and those with mild or asymptomatic COVID-19 infection should delay antenatal visits for 14 days. Women should be classified as low risk or high risk for HDP and adjustments can be accordingly made in the frequency of maternal and fetal surveillance. Patient should be provided detailed instructions concerning their outpatient management but if there is any evidence of disease progression or if acute severe hypertension develop, then prompt hospitalization is suggested.

\author{
Conflict of Interest \\ None declared.
}

\section{References}

1 Roberts JM, August PA, Bakris G, et al; American College of Obstetricians and Gynecologists; Task Force on Hypertension in Pregnancy. Hypertension in pregnancy. Report of the American College of Obstetricians and Gynecologists' task force on hypertension in pregnancy. Obstet Gynecol 2013;122(05):1122-1131

2 Levine RJ, Hauth JC, Curet LB, et al. Trial of calcium to prevent preeclampsia. N Engl J Med 1997;337(02):69-76

3 Hauth JC, Ewell MG, Levine RJ, et al; Calcium for Preeclampsia Prevention Study Group. Pregnancy outcomes in healthy nulliparas who developed hypertension. Obstet Gynecol 2000;95(01): 24-28

4 Sibai BM, Koch MA, Freire S, et al. The impact of prior preeclampsia on the risk of superimposed preeclampsia and other adverse pregnancy outcomes in patients with chronic hypertension. Am J Obstet Gynecol 2011;204(04):345.e1-345.e6

5 Barton JR, Sibai BM. Prediction and prevention of recurrent preeclampsia. Obstet Gynecol 2008;112(2, Pt 1):359-372

6 Pickering TG, Hall JE, Appel LJ, et al; Subcommittee of Professional and Public Education of the American Heart Association Council on High Blood Pressure Research. Recommendations for blood pressure measurement in humans and experimental animals: Part 1: blood pressure measurement in humans: a statement for professionals from the Subcommittee of Professional and Public Education of the American Heart Association Council on High Blood Pressure Research. Hypertension 2005;45(01):142-161

7 Barton JR, Stanziano GJ, Sibai BM. Monitored outpatient management of mild gestational hypertension remote from term. Am J Obstet Gynecol 1994;170(03):765-769

8 Barton JR, Istwan NB, Rhea D, Collins A, Stanziano GJ. Cost-savings analysis of an outpatient management program for women with pregnancy-related hypertensive conditions. Dis Manag 2006;9 (04):236-241

9 Sibai BM, Barton JR. Expectant management of severe preeclampsia remote from term: patient selection, treatment, and delivery indications. Am J Obstet Gynecol 2007;196(06):514.e1-514.e9

10 ACOG Practice Bulletin No. ACOG practice bulletin no. 202: gestational hypertension and preeclampsia. Obstet Gynecol 2019;133(01):e1-e25

11 Sperling JD, Dahlke JD, Huber WJ, Sibai BM. The role of headache in the classification and management of hypertensive disorders in pregnancy. Obstet Gynecol 2015;126(02):297-302

12 Barton JR, Witlin AG, Sibai BM. Management of mild preeclampsia. Clin Obstet Gynecol 1999;42(03):455-469

13 Chahine KM, Sibai BM. Chronic hypertension in pregnancy: new concepts for classification and management. Am J Perinatol 2019; 36(02):161-168

14 Odegård RA, Vatten LJ, Nilsen ST, Salvesen KA, Austgulen R. Preeclampsia and fetal growth. Obstet Gynecol 2000;96(06):950-955

15 Altman D, Carroli G, Duley I, et al; The Magpie Trial Collaborative Group. Do women with pre-eclampsia, and their babies, benefit from magnesium sulphate? The Magpie Trial: a randomised placebo-controlled trial. Lancet 2002;359(9321):1877-1890 